\title{
EVALUACIÓN DE TRES PROGRAMAS DE FERTILIZACIÓN EDÁFICA EN EL CULTIVO DE LA CEBOLLA DE RAMA EN LA CUENCA MEDIA DEL RIO ÓTUN
}

\author{
EVALUATION OF THREE SOIL FERTILIZATION PROGRAMS IN SCALLION CROPS IN \\ THE MID BASIN OF OTUN RIVER
}

Manuel Francisco Polanco-Puerta (D) y Susana Gómez-Posada CuLAC

\begin{abstract}
RESUMEN
Tradicionalmente, el cultivo de cebolla de rama en el municipio de Pereira-Risaralda (cuenca media del rio Otún) ha empleado el estiércol sin compostar, procedente de explotaciones avícolas, como fuente de fertilizante. Esta práctica genera un impacto negativo tanto al cultivo como al medio ambiente ya que favorece la aparición de plagas y enfermedades, la contaminación de suelos y aguas y la proliferación de mosca doméstica que afecta la salud de los pobladores de la zona. Este trabajo se realizó en parcelas de productores, bajo la estrategia de investigación participativa, y su objetivo fue evaluar el efecto de tres programas de fertilización alternativa, empleando como testigo la fertilización tradicional con gallinaza sin compostar. Se utilizó un diseño experimental de Bloques Completos al Azar con tres repeticiones y evaluación en tres ciclos continuos de producción. De acuerdo con los resultados, la aplicación de gallinaza sin compostar puede ser reemplazada con la implementación de un programa adecuado de fertirriego y obtener niveles de producción similares (20,5 y 19,1 tha ${ }^{-1}$ respectivamente). La rentabilidad del cultivo fue mayor con el tratamiento de fertilizantes químicos sólidos aplicados al suelo $(34,0 \%)$ respecto a la aplicación de gallinaza sin compostar $(23,7$ \%). La rentabilidad del tratamiento fertirriego fue $19,1 \%$ y estuvo afectada por los altos costos de su implementación. El tratamiento con abono compostado presentó la producción y la rentabilidad más bajas con 18,7 t ha $^{-1}$ y $16,8 \%$, respectivamente.
\end{abstract}

PALABRAS CLAVE: fertigación; abonos orgánicos; compostados; productividad; rendimiento

\begin{abstract}
Traditionally in scallion cultivation in the middle basin of the Otún River in the municipality of Pereira Risaralda, unmanufactured manure from poultry farms has been used as a fertilizer source. This practice has a negative impact on both the crop and the environment, since it favors the appearance of pests and diseases in the crop, the contamination of soils and water and the proliferation of domestic fly that affects the health of the residents of the area. This work was carried out in producer plots under the participative research strategy and its objective was to evaluate the effect of three fertilization programs, using as a control the application of uncooked hen. A randomized complete block experimental design with three replications and evaluation in three continuous cycles of production was used. According to the results, the application of uncooked hen can be replaced with the implementation of an adequate ferti irrigation program and obtain the same production levels, 20.5 and 19.1 $t \mathrm{th}^{-1}$ respectively. With respect to the yield of the crop, this was greater with the treatment of solid chemical fertilizers applied to the soil $34 \%$ compared to $23.7 \%$ with the application of poultry without composting. The profitability of the fertigation treatment was affected by the high costs of its implementation $19.1 \%$. The treatment with compost fertilizer presented a low production $18.7 \mathrm{t} \mathrm{ha}^{-1}$ and the lowest profitability $16.8 \%$.
\end{abstract}

KEY WORDS: fertigation; organic manure; composted; productivity; crop yield

Dirección de los autores:

Docente Universidad Nacional Abierta y a Distancia UNAD-CCAV Eje Cafetero. Carrera 23 Diagonal 25 F Casas de Milán, Dosquebradas Risaralda, Colombia. Teléfono (6)3116599 Ext. 113; e-mail: manuel.polanco@unad.edu.co ORCID: 0000-0002-4810-0081 I.A. Esp. M.Sc. PhD. (M.F.P.P.); e-mail: susana.gomez@unad.edu.co I.A. M.Sc. (S.G.P.). 


\section{INTRODUCCIÓN}

La gallinaza fresca es la principal fuente fertilizante que utilizan los productores de cebolla de rama tanto en la cuenca media del río Otún como en la mayoría de zonas productoras del país y su aplicación se hace en forma superficial, alrededor de la planta. Si bien, es una fuente rica en nutrientes minerales que favorece el desarrollo de las plantas, al no estar compostada, provoca un aumento en la incidencia de plagas y enfermedades limitantes que van en detrimento del rendimiento del cultivo, lo que obliga al agricultor a utilizar una mayor cantidad de agroquímicos para su control incrementando así los costos de producción.

Además, la aplicación de gallinaza cruda a los cultivos genera la proliferación de mosca doméstica en poblaciones tan altas, que ha sido considerada como un problema de salud pública para la comunidad circunvecina (CORPOICA, 1993; Castellanos, 1999; Agúdelo, 2007; CARDER, 2008; Guzmán y Palacios, 2008).

Esta situación ha venido siendo evidenciada por los productores, quienes ante la eminente aplicación de la reglamentación ICA de la circular de fecha 1 de junio de 2007 en donde se prohíbe la utilización de la gallinaza fresca como abono, han tenido que considerar el uso de otras fuentes de fertilización orgánicas e inorgánicas que ofrezcan la posibilidad de mejorar o al menos mantener la productividad de sus cultivos. Sin embargo hasta el momento, los planes de fertilización alternativa han sido implementados sin tener en cuenta los análisis de suelos y los requerimientos nutricionales del cultivo, dando como resultado producciones más bajas a las obtenidas con el manejo tradicional.

Las enmiendas orgánicas compostadas si bien, son la forma adecuada de proveer materia orgánica a los cultivos, presentan un porcentaje menor de nitrógeno disponible comparado con el de la gallinaza fresca, por lo cual se hace necesario buscar otras alternativas que complementen la nutrición de los cultivos y que vayan en beneficio tanto de la producción como del medio ambiente (Guzman y Palacios, 2008).

De los diferentes sistemas de riego, el riego por goteo es el que cuenta con las mayores eficiencias de aplicación, y en general las pérdidas de agua pueden reducirse sustancialmente cuando el sistema está bien diseñado, operado y mantenido (Bralts et al., 1987). El riego por goteo es un sistema que utiliza mangueras de presión con el agua gota a gota, lo más cerca a la raíz de la planta, formando un bulbo de humectación con humedad constante (Silva et al., 2013). La uniformidad que se logra con este sistema de riego, permite maximizar el uso del agua, limitar las pérdidas, mejorar la fertilización cuando se realiza vía fertirriego y aminora los impactos ambientales asociados con la contaminación de las aguas subterráneas (Hagin et al., 2002).

El fertirriego, empleando el sistema de riego por goteo, ha sido considerado como una de las mejores formas de fertilización, ya que los nutrientes son entregados en forma fraccionada de acuerdo a los requerimientos del cultivo en cada etapa de desarrollo y en formas químicas solubles de rápida asimilación por la planta.

Específicamente en Risaralda, municipio de Pereira, Vereda La Bella, sitio en donde se desarrolló este experimento, los suelos dedicados a la siembra de cebolla de rama son francos, color negro y con alta retención de humedad, procedentes de cenizas volcánicas, con alta fertilidad y con pendientes inferiores al $70 \%$ (Machado et al., 1983).

Dando respuesta a la problemática anterior, en éste ensayo se probaron tres programas de fertilización para el cultivo de la cebolla de rama (incluida la fertirrigación) y se evaluó el impacto de cada uno de ellos sobre la producción, utilizando como indicadores parámetros de desarrollo del cultivo como largo y diámetro del pseudotallo y rendimiento del cultivo (t ha $\left.{ }^{-1}\right)$. Por lo tanto, el objetivo principal de esta investigación fue proponer un programa de fertilización para el cultivo de la Cebolla de rama que permita la obtención de rendimientos aceptables en la producción y la disminución en la incidencia de plagas y enfermedades del cultivo sin detrimento de la rentabilidad del ejercicio productivo, convirtiéndose en un referente técnico y un aporte a la solución de la problemática actual que enfrentan los agricultores de la cuenca media del rio Otún.

\section{MATERIALES Y MÉTODOS}

\section{Localización}

Los tratamientos se evaluaron en tres localidades diferentes ubicadas en la cuenca media del rio Otún, correspondientes a las fincas Guacamal en Corregimiento de la Florida a $1695 \mathrm{~m}$ de altura, Lucitania y la Esmeraldita en el Corregimiento de La Bella, a $1827 \mathrm{~m}$ y $1866 \mathrm{~m}$ de altura respectivamente; la temperatura oscila entre los 14 a $18{ }^{\circ} \mathrm{C}$, humedad relativa promedia del 85 
\% y con una precipitación de 2462 a 2563 mm anuales. Los meses de mayor precipitación son los periodos de marzo a junio y de septiembre a noviembre. Los periodos secos se presentan en los meses de junio a agosto y de diciembre a febrero. Es una zona que permanece con mucha nubosidad por lo que el promedio de horas de brillo solar es de 4 horas día (Jaramillo, 1997).

Las evaluaciones se realizaron durante tres ciclos de cultivo (épocas diferentes de evaluación) entre los meses de enero a julio de 2014 y consistieron en los diferentes programas de fertilización calculados con base en los resultados de los análisis de suelos y los requerimientos nutricionales para cebolla junca, propuestos por Jaramillo et al. (1996), así:

T1 Sistema tradicional: Fertilización orgánica con gallinaza sin compostar $\left(14 \mathrm{t} \mathrm{ha}^{-1}=400 \mathrm{gr} /\right.$ sitio $)+$ Fertilización foliar con Tecnoverde ${ }^{\circledR} 1$ / ha.

T2 Fertilización orgánica con Abono compostado (14 tha ${ }^{-1}=$ $400 \mathrm{gr}$ / sitio) + Fertilización foliar con Bioagro Triple A 11 / ha.

T3 Fertilización química vía Fertiriego microcontrolado, con fertilizante complejo soluble (Multifeed ${ }^{\circledR} 20-20-20+$ elementos menores $=10 \mathrm{~g} /$ sitio $=350 \mathrm{~kg} / \mathrm{ha}$ ).

T4 Fertilización química con fertilizante sólido aplicado al suelo $\left(\right.$ Abotek $^{\circledast} 20 \mathrm{~g} /$ sitio $\left.=700 \mathrm{~kg} \mathrm{ha}^{-1}\right)+$ elementos menores en inyección al suelo. Aplicados 20 días después del deshije.

El material genético utilizado en las evaluaciones, fue la cultivariedad de cebolla de rama (Alliun fistulosum) "La Pereirana", la cual ocupa 48 \% del área cultivada en cebolla en la zona y que ha mostrado una amplia adaptación a las condiciones locales.

Los tratamientos de fertilización se implementaron en cultivos tradicionales después del deshije de la cebolla. Se realizaron todas las prácticas culturales de manejo como enterrado de arvenses, aporques y manejo de los problemas fitosanitarios, de la manera tradicional e igual para todos los tratamientos. Se realizó análisis físico químico de suelo para cada una de las localidades en el laboratorio de suelos de la Universidad Tecnológica de Pereira (Anexo I)*.

Los caracteres evaluados fueron: Longitud del seudotallo (LT): Diámetro del seudotallo (DT); Peso de seudotallo (PT); Número de seudotallos por sitio (NTS); Peso de seudotallos por sitio (PTS), relación seudotallo-hoja (RTH); Longitud de las hojas (LH), Diámetro de hoja (DH); Longitud de la raíz (LR)

\section{Diseño experimental}

Se estableció un diseño experimental en Bloques Completos al Azar (BCA), con cuatro tratamientos, y tres repeticiones, con un total de 12 unidades experimentales. Cada tratamiento consto de 3 surcos, separados 80 $\mathrm{cm}$ por 8 metros de largo, sembrados a $30 \mathrm{~cm}$ entre sitios, quedando establecidas parcelas experimentales de $240 \mathrm{~m}^{2}$, con una población total de 1000 sitios y aproximadamente 6000 plantas para el experimento. Los resultados se analizaron con el programa SAS ${ }^{\circledR}$ (Statistical Analysis System. versión 9.2) mediante análisis de varianza y prueba de comparación de medias de Tukey para las variables estadísticamente diferentes. Adicionalmente, se efectuaron análisis de correlación lineal de Pearson entre las variables de respuesta agrupando todos los datos de las tres localidades, para cada uno de los caracteres morfoagronómicos evaluados.

Se realizó el análisis de los costos de fertilización de cada uno de los tratamientos como criterio de decisión para determinar su rentabilidad, considerando todos los rubros que intervienen en el proceso de productivo de la cebolla de rama en zona y clasificándolos en costos directos e indirectos (Anexo II)*.

\section{RESULTADOS}

Los resultados e interpretación de los análisis del suelo para los tres predios se presentan en la tabla 1. Mostraron, de manera general, una textura franca, $\mathrm{pH}$ moderadamente ácido, altos contenidos de materia orgánica, altos niveles de fosforo, potasio y calcio, magnesio bajo, y altos niveles de cobre, manganeso y zinc.

Se realizó el análisis de varianza para cada fuente de variación (localidades, tratamientos y épocas) y la interacción entre ellas de acuerdo al modelo establecido de BCA. El efecto de los tratamientos fue significativo para las variables número de seudotallos, longitud de seudotallos y longitud de hojas. No se presentaron diferencias significativas para las variables diámetro de seudotallo DT, número de hojas por tallo NHT, longitud de raíz LR, y el peso total de la planta.

*Archivo disponible en la página web de la Revista Intropica. 
Tabla 1. Análisis físico químico de suelo de los predios evaluados.

\begin{tabular}{|c|c|c|c|c|c|c|}
\hline \multirow{3}{*}{ Descripción } & \multicolumn{6}{|c|}{ PREDIOS } \\
\hline & \multicolumn{2}{|c|}{ Esmeraldita } & \multirow{2}{*}{$\begin{array}{c}\text { Lucitanía } \\
\text { Encontrado }\end{array}$} & \multicolumn{3}{|c|}{ Guacamal } \\
\hline & Encontrado & Interpretación & & Interpretación & Encontrado & Interpretación \\
\hline Textura & Franco & Adecuado & Franco & Adecuado & Franco & Adecuado \\
\hline $\begin{array}{l}\text { Conductividad } \\
\text { eléctrica }\end{array}$ & 0,108 & & 0,103 & & 0,11 & \\
\hline $\mathrm{CIC}$ & $* * *$ & & $* * *$ & & $* * *$ & \\
\hline $\mathrm{pH}$ & 6,1 & $\begin{array}{l}\text { Ligeramente } \\
\text { ácido }\end{array}$ & 5,8 & $\begin{array}{l}\text { Moderadamente } \\
\text { ácido }\end{array}$ & 6,2 & $\begin{array}{l}\text { Ligeramente } \\
\text { ácido }\end{array}$ \\
\hline $\begin{array}{l}\text { Materia } \\
\text { Orgánica (\%) }\end{array}$ & 10,2 & Alto & 10,6 & Alto & 11,2 & Alto \\
\hline Nitrógeno & 0,41 & Alto & 0,42 & Alto & 0,48 & Alto \\
\hline Aluminio & $* * *$ & & $* * *$ & & $* * *$ & \\
\hline $\begin{array}{l}\text { Fosforo (Bray II) } \\
\text { (ppm) }\end{array}$ & 48 & Alto & 51 & Alto & 56 & Alto \\
\hline $\begin{array}{l}\text { Potasio (meq / } \\
100 \mathrm{~g} \text { ) }\end{array}$ & 0,45 & Alto & 0,50 & Alto & 0,60 & Alto \\
\hline $\begin{array}{l}\text { Calcio (meq / } \\
100 \mathrm{~g})\end{array}$ & 6,8 & Alto & 7,3 & Alto & 7,1 & Alto \\
\hline $\begin{array}{l}\text { Magnesio (meq } \\
\text { / } 100 \mathrm{~g} \text { ) }\end{array}$ & 1,4 & Bajo & 1,6 & Bajo & 1,5 & Bajo \\
\hline Hierro (ppm) & 194 & Alto & 1,90 & Alto & 1,74 & Alto \\
\hline $\begin{array}{l}\text { Manganeso } \\
(\mathrm{ppm})\end{array}$ & 18 & Alto & 15 & Alto & 12 & Alto \\
\hline Zinc (ppm) & 22 & Alto & 27 & Alto & 22 & Alto \\
\hline Cobre(ppm) & 17 & Alto & 19 & Alto & 14 & Alto \\
\hline
\end{tabular}

PTP (Tabla 2). El efecto de las diferentes épocas de evaluación (ciclos de cultivo) también presentó una influencia altamente significativa sobre todas las variables. En la tabla 2 también se puede apreciar que las localidades tienen un efecto altamente significativo sobre todas las variables de respuesta, con excepción en la longitud de los seudotallos debidos posiblemente a efectos de microclima y contenidos nutricionales del suelo, que presentaron leves diferencias. 
El efecto de la interacción localidad por tratamiento, fue altamente significativo para la gran mayoría de las variables evaluadas menos para las variables diámetro de los seudotallos y peso total de la planta. $\mathrm{Al}$ analizar la interacción entre épocas y tratamientos, se encontró que este, no tuvo un efecto significativo sobre las variables de respuesta número de seudotallos por sitio, número de hojas por tallo, longitud de la hoja, longitud de la raíz y sobre el peso total de la planta, pero si tuvo un efecto altamente significativo sobre la longitud y diámetro de los seudotallos. La interacción entre localidad, épocas y tratamientos, no presentó diferencias significativas sobre las variables de respuesta, con excepción del número de hojas por tallo y la longitud de la raíz, en las que el efecto fue altamente significativo (Tabla 2).

Tabla 2. Análisis de varianza de los caracteres morfoagronómicos cuantitativos evaluados para determinar los efectos de fertilizante en el cultivo de cebolla rama en todas las localidades. Los valores corresponden a los cuadrados medios. NTS: número de tallos por sitio; LT: longitud de seudotallos (cm); DT: diámetro de los seudotallos (cm); NHT: número de hojas por tallo; LH: longitud de las hojas (cm); LR: longitud de la raíz (cm); PTP: peso total por planta (g). ${ }^{*} \mathrm{P}<0,01 ;{ }^{* *} \mathrm{P}<0,005$ $\% ; \mathrm{ns}=$ no significativo.

\begin{tabular}{lcccccccc}
\hline \multirow{2}{*}{ FV } & \multicolumn{1}{c}{ GL } & \multicolumn{7}{c}{ CM } \\
\cline { 2 - 8 } & NTS & LT & DT & NHT & LH & LR & PTP \\
\hline Localidad & 2 & $823,22^{* *}$ & $0,22 \mathrm{~ns}$ & $0,64^{* *}$ & $2389,30^{* *}$ & $2584,20^{* *}$ & $735,13^{* *}$ & $265,00^{* *}$ \\
Época & 2 & $88,73^{* *}$ & $226,36^{* *}$ & $0,09^{* *}$ & $2034,66^{* *}$ & $3532,99^{* *}$ & $555,18^{* *}$ & $1983,70^{* *}$ \\
Localidad x Época & 4 & $426,10^{* *}$ & $245,14^{* *}$ & $0,51^{* *}$ & $2164,48^{* *}$ & $2636,67^{* *}$ & $96,55^{* *}$ & $1523,71^{* *}$ \\
Rep. (Loc. x Época) & 18 & $8,20 \mathrm{~ns}$ & $63,45 \mathrm{~ns}$ & $0,02 \mathrm{~ns}$ & $1,44 \mathrm{~ns}$ & $15,19^{* *}$ & $2,70 \mathrm{~ns}$ & $65,37 \mathrm{~ns}$ \\
Tratamiento & 3 & $32,22^{* *}$ & $2,50^{* *}$ & $0,04 \mathrm{~ns}$ & $4,49 \mathrm{~ns}$ & $27,09^{* *}$ & $12,46 \mathrm{~ns}$ & $168,77 \mathrm{~ns}$ \\
Loc. x Tratamiento. & 6 & $45,06^{* *}$ & $54,72^{* *}$ & $0,01 \mathrm{~ns}$ & $4,33^{* *}$ & $17,35^{* *}$ & $20,25^{* *}$ & $69,15 \mathrm{~ns}$ \\
Época x Tratamiento. & 6 & $11,43 \mathrm{~ns}$ & $62,82^{* *}$ & $0,06^{* *}$ & $2,74 \mathrm{~ns}$ & $5,36 \mathrm{~ns}$ & $4,93 \mathrm{~ns}$ & $105,96 \mathrm{~ns}$ \\
Loc. x Época x Trat. & 12 & $12,39 \mathrm{~ns}$ & $60,42 \mathrm{~ns}$ & $0,02 \mathrm{~ns}$ & $4,03^{* *}$ & $6,59 \mathrm{~ns}$ & $14,01^{* *}$ & $58,81 \mathrm{~ns}$ \\
Promedio & & 23,04 & 18,61 & 1,79 & 10,89 & 45,99 & 14,60 & 54,87 \\
CV \% & & 10,42 & 9,54 & 6,45 & 10,70 & 4,78 & 12,81 & 12,34 \\
\hline
\end{tabular}

Se encontró que la localidad Guacamal presentó los mejores promedios para las variables número de tallos por sitio 28,23 diámetro de los seudotallos $1,95 \mathrm{~cm}$, número de hojas por tallo 20,30 y peso total por planta $57,08 \mathrm{~g}$ y los promedios más bajos para las variables longitud y diámetro de hojas. El valor de la longitud de los seudotallos fue igual para todas las localidades (Tabla 3). En la localidad Lucitania, se presentó el mejor comportamiento de las plantas, en cuanto a longitud de las hojas y peso total de las plantas y valores intermedios para las demás variables y la localidad La Esmeraldita, presentó el mejor comportamiento de la cebolla para la variable diámetro de la hoja y fue la localidad con los valores más bajos para las demás variables de respuesta (Tabla 3).
En cuanto a la interacción entre los tratamientos, localidades y épocas sobre las variables de respuesta, los resultados evidencian que la mejor respuesta de las plantas de cebolla para cada una de las variables ocurre con la aplicación de gallinaza sin compostar, aunque los valores en la longitud y diámetro de los seudotallos fueron iguales para todos los tratamientos. Un segundo lugar es para el tratamiento con fertirriego, que presenta valores iguales al tratamiento con gallinaza, en las variables de: longitud de seudotallos, diámetro de los seudotallos y en el diámetro de las hojas, y valores muy cercanos en las variables número de hojas por seudotallos y peso total cosechado. En un tercer y cuarto lugar se pueden ubicar los tratamientos T2 y T4 de fertilización orgánica con gallinaza compostada y fertilización con fertilizante 
químico completo al suelo, que presentan valores en las variables de respuesta muy parecidos y en donde no hubo diferencias significativas entre ellos en términos de rendimiento de cultivo (Tabla 4). Los valores de peso por planta, transformados a producción de toneladas por hectárea, se pueden apreciar en la figura 1.

Tabla 3. Promedio de 36 mediciones de los caracteres morfoagronómicos cuantitativos evaluados por localidad. NTS: número de tallos por sitio; LT: longitud de seudotallos (cm); DT: diámetro de los seudotallos (cm); NHT: número de hojas por tallo; LH: longitud de las hojas (cm); DH: diámetro de las hojas (cm); PTP: peso total por planta (g). Valores entre columnas seguido por letras diferentes son estadísticamente diferentes $(\mathrm{P}<0,05)$ de acuerdo a la prueba de separación de medias de Tukey.

\begin{tabular}{lccccccc}
\hline & \multicolumn{7}{c}{ CARÁCTER MORFOAGRONÓMICO } \\
\cline { 2 - 7 } \multicolumn{1}{c}{ LOCALIDAD } & NTS & LT & DT & NHT & LH & DH & PTP \\
\hline Guacamal & $28,23 \mathrm{a}$ & $18,59 \mathrm{a}$ & $1,95 \mathrm{a}$ & $20,30 \mathrm{a}$ & $36,57 \mathrm{c}$ & $9,66 \mathrm{c}$ & $57,08 \mathrm{a}$ \\
Lucitanía & $22,11 \mathrm{~b}$ & $18,59 \mathrm{a}$ & $1,71 \mathrm{~b}$ & $6,01 \mathrm{~b}$ & $52,99 \mathrm{a}$ & $13,82 \mathrm{~b}$ & $55,69 \mathrm{ba}$ \\
Esmeraldita & $18,80 \mathrm{c}$ & $18,67 \mathrm{a}$ & $1,73 \mathrm{~b}$ & $6,37 \mathrm{~b}$ & $48,41 \mathrm{~b}$ & $18,69 \mathrm{a}$ & $51,84 \mathrm{~b}$ \\
\hline
\end{tabular}

Tabla 4. Promedio de 27 mediciones de los caracteres morfoagronómicos cuantitativos evaluados por tratamiento. Valores entre columnas seguido por letras diferentes son estadísticamente diferentes $(\mathrm{P}<0,05)$ de acuerdo a la prueba de separación de medias de Tukey. Abreviaturas como en la tabla 3.

\begin{tabular}{lccccccc}
\hline \multirow{2}{*}{ TRATAMIENTO } & \multicolumn{7}{c}{ DESCRIPTOR* $^{*}$} \\
\cline { 2 - 8 } & NTS & LT & DT & NHT & LH & DH & PTP \\
\hline Gallinaza & $24,5 \mathrm{a}$ & $18,4 \mathrm{a}$ & $1,82 \mathrm{a}$ & $11,3 \mathrm{a}$ & $47,4 \mathrm{a}$ & $13,8 \mathrm{ba}$ & $58,5 \mathrm{a}$ \\
Fertirriego & $21,8 \mathrm{~b}$ & $18,7 \mathrm{a}$ & $1,82 \mathrm{a}$ & $10,6 \mathrm{ba}$ & $45,1 \mathrm{~b}$ & $15,1 \mathrm{a}$ & $54,5 \mathrm{ba}$ \\
Químico & $22,9 \mathrm{ba}$ & $18,8 \mathrm{a}$ & $1,74 \mathrm{a}$ & $10,4 \mathrm{~b}$ & $45,5 \mathrm{a}$ & $13,7 \mathrm{~b}$ & $52,9 \mathrm{~b}$ \\
Compost & $22,6 \mathrm{ba}$ & $18,5 \mathrm{a}$ & $1,78 \mathrm{a}$ & $11,1 \mathrm{ba}$ & $45,8 \mathrm{ba}$ & $13,6 \mathrm{~b}$ & $53,5 \mathrm{~b}$ \\
\hline
\end{tabular}

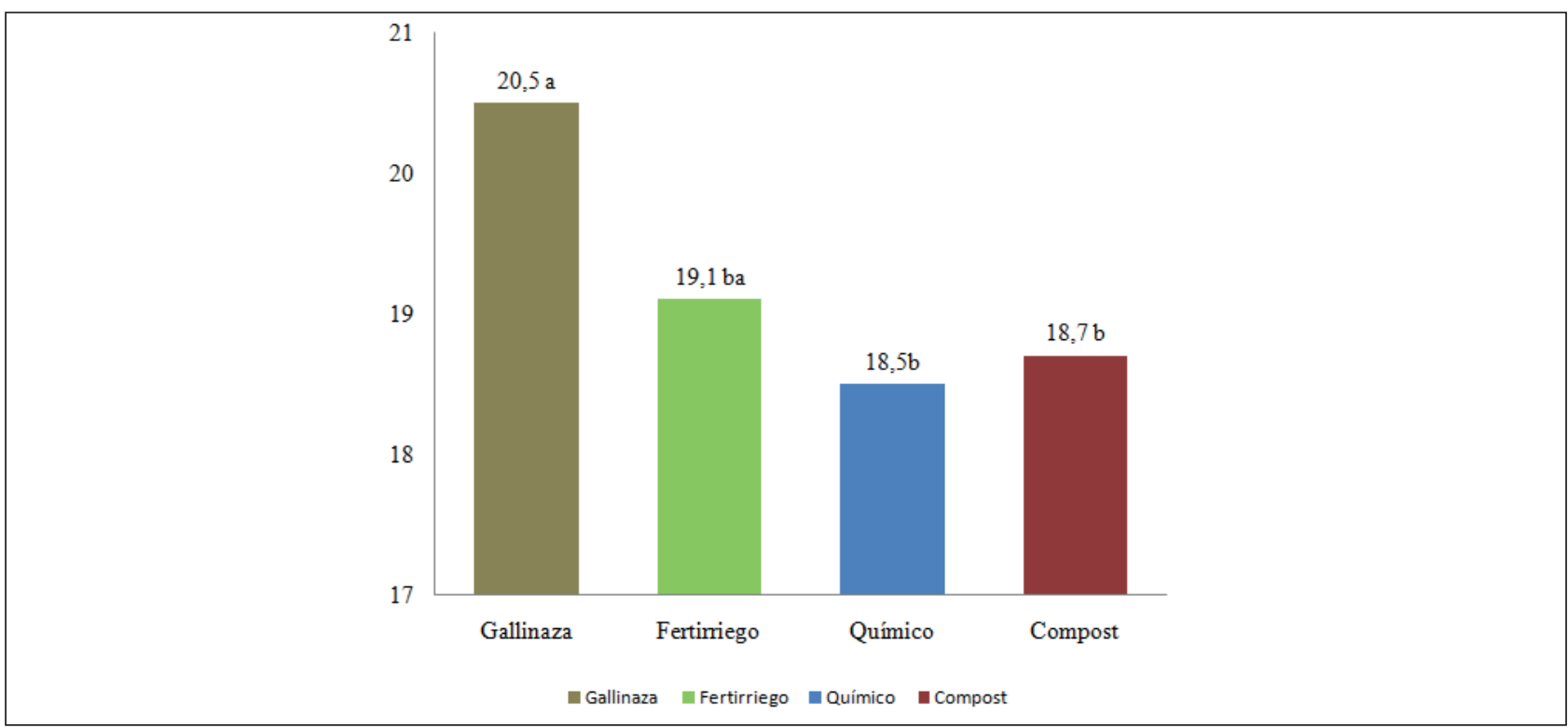

Figura 1. Producción de cebolla de rama por cada tratamiento ( $\mathrm{t} \mathrm{ha} \mathrm{a}^{-1}$ ). 
El análisis de correlación entre variables de respuesta resultó positiva y altamente significativa entre el número de seudotallos por sitio y número de hojas por tallo ( $\mathrm{r}$ $=0,445)$, el número de seudotallos cosechados ( $\mathrm{r}=$ $0,999)$ y el peso total cosechado $(r=0,876)$ y negativa altamente significativa entre el número de seudotallos cosechados con la longitud de las hojas ( $\mathrm{r}=-0,371$ ) y con la longitud de la raíz $(r=-0,536)$. La longitud de los seudotallos, presentó correlación altamente significativa con las variables de longitud de hojas, longitud de raíz y peso de la planta $(\mathrm{r}=0,582 ; 0,403$; y 0,342 respectivamente) y su correlación fue negativa y altamente significativa con el número de hojas por seudotallo $(r=-0,547)$. El diámetro de los seudotallos presentó correlación altamente significativa con las variables número de hojas por seudotallo $(\mathrm{r}=0,470)$, peso de la planta $(\mathrm{r}=0,703)$ y peso total cosechado $(\mathrm{r}=0,373)$ y correlación negativa y altamente significativa con la longitud de las hojas $(\mathrm{r}=-0,354)$. Al correlacionar el número de hojas por seudotallo, contra las demás variables de respuesta, se encontró relación altamente significativa con el número de tallos a cosecha $(r=0,445)$ y con el peso total cosechado ( $r$
$=0,349)$ y correlación negativa altamente significativa con la longitud de las hojas y la longitud de la raíz ( $r=-0,961 ;-0,754$ respectivamente). La longitud de las hojas, también presentó correlación positiva y altamente significativa con la longitud de la raíz ( $\mathrm{r}=$ $0,689)$ y correlación negativa y altamente significativa con el número de tallos a cosecha $(\mathrm{r}=-0,371)$. La correlación del número de tallos a cosecha con el peso total cosechado fue positiva y altamente significativa ( $\mathrm{r}$ $=0,876$ ) (Tabla 5).

Los resultados del análisis de costos para cada tratamiento se muestran en la tabla 6 y fueron calculados de acuerdo a los ítems reportados en el anexo II $^{*}$. Se pudo evidenciar que la mayor rentabilidad del sistema productivo se logra con la aplicación de fertilizante químico completo, a pesar de que el nivel de producción sea el más bajo. La rentabilidad más baja del cultivo, se obtuvo bajo el programa de fertilización con gallinaza compostada debida principalmente a los altos costos de la mano de obra.

*Archivo disponible en la página web de la Revista Intropica.

Tabla 5. Correlaciones entre caracteres morfoagronómicos cuantitativos evaluados en cuatro tratamiento de fertilización en el cultivo de cebolla rama efectuado en tres localidades, durante tres épocas diferentes. NTS: número de tallos por sitio; LT: longitud de seudotallos (cm); DT: diámetro de los seudotallos $(\mathrm{cm})$; NHT: número de hojas por tallo; LH: longitud de las hojas (cm) LR: longitud de la raíz (cm); PTP: peso total por planta (g); NTC: número de tallos en cosecha; PTC: peso total de la cosecha. ${ }^{*} \mathrm{P}<0,01{ }^{* *} \mathrm{P}<0,005$; ns: no significativo.

\begin{tabular}{|c|c|c|c|c|c|c|c|c|c|}
\hline \multicolumn{10}{|c|}{ Coeficiente de correlación de Pearson, $\mathrm{N}=108$} \\
\hline DESCRIPTOR & NTS & LT & DT & NHT & LH & LR & PTP & NTC & PTC \\
\hline NTS & 1,000 & $-0,230 \mathrm{~ns}$ & $0,071 \mathrm{~ns}$ & $0,445^{* *}$ & $-0,371^{* *}$ & $-0,536^{* *}$ & $-0,110 \mathrm{~ns}$ & $0,999 * *$ & $0,876^{* *}$ \\
\hline LT & & 1,000 & $-0,030 \mathrm{~ns}$ & $-0,547^{* *}$ & $0,582 * *$ & $0,403^{* *}$ & $0,341^{* *}$ & $-0,229 n s$ & $-0,045 \mathrm{~ns}$ \\
\hline DT & & & 1,000 & $0,470 * *$ & $-0,354^{* *}$ & $-0,227 n s$ & $0,703^{* *}$ & $0,072 \mathrm{~ns}$ & $0,373^{* *}$ \\
\hline NHT & & & & 1,000 & $-0,961 * *$ & $-0,754^{* *}$ & $-0,051 \mathrm{~ns}$ & $0,445^{* *}$ & $0,349^{* *}$ \\
\hline $\mathrm{LH}$ & & & & & 1,000 & $0,689^{* *}$ & $0,218 \mathrm{~ns}$ & $-0,371^{* *}$ & $-0,207 \mathrm{~ns}$ \\
\hline LR & & & & & & 1,000 & $0,152 \mathrm{~ns}$ & $-0,536 \mathrm{~ns}$ & $-0,403 n s$ \\
\hline РTP & & & & & & & 1,000 & $-0,110 \mathrm{~ns}$ & $0,349 \mathrm{~ns}$ \\
\hline NTC & & & & & & & & 1,000 & $0,876^{* *}$ \\
\hline PTC & & & & & & & & & 1,000 \\
\hline
\end{tabular}


Tabla 6. Análisis de rentabilidad de cada tratamiento de fertilización en función de la productividad ( ha $^{-1}$ ) en el cultivo de la cebolla de rama.

\begin{tabular}{lcc}
\hline \multicolumn{1}{c}{ Tratamiento } & Producción $\mathbf{~ t ~ h a ~}^{-1}$ & Rentabilidad \% \\
\hline Gallinaza sin compostar & $20,5 \mathrm{a}$ & 23,7 \\
Fertirriego & $19,1 \mathrm{ba}$ & 19,1 \\
Abono compostado & $18,7 \mathrm{~b}$ & 16,8 \\
Fertilizante Químico completo & $18,5 \mathrm{~b}$ & 34,0 \\
\hline
\end{tabular}

\section{DISCUSIÓN}

Las diferencias encontradas entre los tratamientos (Tablas 2 y 4), indican que los programas de fertilización tienen gran influencia en la respuesta de producción de cultivo de la cebolla de rama y en donde la aplicación de gallinaza sin compostar tiene un efecto positivo mayor sobre todas las variables de respuesta evaluadas, comparada con los tratamientos de fertilización química, el abono compostado y el fertirriego. Esto se explica en el sentido que la gallinaza sin compostar, al ser aplicada al suelo comienza un proceso normal de descomposición en donde libera rápidamente ácidos fúlvicos, que proveen a las plantas nitrógeno, fósforo y micro nutrientes de forma casi inmediata y posteriormente durante el proceso de mineralización sigue liberando nutrientes de forma más lenta y oportuna durante todo el ciclo de cultivo, especialmente en la fase de crecimiento, en el que el contenido del nitrógeno incrementa el diámetro del tallo, y la longitud de los tallos (Machado et al, 1983; Guerrero, 1996).

En el caso de los fertilizantes químicos compuestos NPK utilizados por los productores de la zona se producen grandes perdidas de nitrógeno por volatilización, de fósforo por fijación y de potasio por lixiviación, y en el caso de los abonos compostados, los contenidos de nitrogeno y de otros nutrientes son más bajos y su liberación depende del proceso lento de mineralización de la materia orgánica en donde los nutrientes, especialmente el nitrógeno, es inmovilizado temporalmente por los microorganismos del suelo y por tanto no se encuentra disponible de manera inmediata en forma asimilable para las planta, además de que una buena parte de él se volatiliza en forma de NH3-N dado que la materia orgánica no es incorporada al suelo sino que se aplica de manera superficial.
Según Jaramillo et al. (1996), considerando la fertilización que realizan los cultivadores de cebolla del municipio de Aquitania, la aplicación de abonos orgánicos para el cultivo de cebolla, debe hacerse sólo en suelos con contenidos bajos de materia orgánica (menos del $4 \%$ ) y en dosis moderada (4-8 t ha-1), se debe corregir la acidez del suelo, pues la cebolla crece mejor en pH entre 6,0 a 7,0, por lo que se requiere la aplicación de cal dolomítica o calfos en dosis de 1 a 4 toneladas por hectáreas (ICA, 1983), y aplicar 10-30-10 en dosis de $500 \mathrm{~kg} \mathrm{ha}^{-1}$. Para efectos del experimento se empleó la cantidad de gallinaza y abono compostado que aplican los productores de $14 \mathrm{t} \mathrm{ha}^{-1}$ por ciclo, y 700 kg de Abotek $^{\circledR}$ (15-4-23-4), considerando los altos contenidos del NPK en el suelo.

La evaluación del efecto de las épocas en la que se realizó el ensayo (ciclos de cultivo) (Tabla 2), presentó diferencias altamente significativas sobre la respuesta de las variables evaluadas. Esto indica que hay un efecto ambiental sobre la mayoría de las variables, máxime cuando estas variables, son caracteres cuantitativos manejados por muchos genes, tal como lo señalan Vallejo y Estrada (2002).

La prueba de Tukey (Tabla 3), ayudó a determinar que en la localidad Guacamal se presentaron los mejores promedios para las variables número de tallos por sitio 28,23 , diámetro de los seudotallos $1,95 \mathrm{~cm}$, número de hojas por tallo 20,30 y el peso total por planta 57,08 g., y los valores más bajos para las variables longitud y diámetro de las hojas. En la localidad Lucitania, se presentó el mejor comportamiento de las plantas en cuanto a longitud de las hojas y peso total de las plantas. La localidad La Esmeraldita, presento el mejor comportamiento de la cebolla para la variable 
diámetro de la hoja y fue la localidad con los valores más bajos para las demás variables de evaluación. Estos comportamientos diferenciales de las características evaluadas en la cebolla para cada lugar, puede ser explicada por las diferentes condiciones agroecológicas y de suelo en cada una de las localidades (Tabla 1), que influyen sobre el crecimiento y desarrollo de las plantas.

De los resultados del análisis de correlación (Tabla 5), cuando se analizaron en conjunto las tres localidades, los tratamientos, las épocas y las repeticiones, lo más importante de resaltar es que existe una correlación altamente significativa entre el número de tallos por sitio y la producción total del cultivo, esta condición aunque positiva en términos de volumen de producción, resulta no muy provechosa en términos de calidad, pues este mayor número de tallos por sitio, resulta en un menor diámetro de estos, lo cual es una condición de baja calidad. También se presenta una correlación altamente positiva entre la longitud de los seudotallos y la longitud de las hojas y de la raíz.

El diámetro de los tallos está altamente correlacionado con el número de hojas, pero el diámetro del tallo se disminuye a media que la longitud de las hojas se aumenta, por lo que se hace evidente que el diámetro y el número de hojas depende de la variedad y de las condiciones ambientales y nutricionales del suelo, como lo manifiestan Benavides y Siles (1990).

La ausencia de variación en el diámetro de los seudotallos por efecto de los tratamientos puede significar que es un carácter poco influenciado por el ambiente y manejado por pocos genes, pues esta situación se repitió en las tres localidades y durante las tres épocas de evaluación. Sin embargo se evidenció una leve diferencia en el tratamiento con gallinaza sin compostar, lo que puede significar que al mejorar los niveles de fertilización en los otros tratamientos, se podría aumentar el valor de ésta variable.

La variable producción, tuvo una correlación altamente significativa con el número de seudotallos por sitio y con la longitud y número de hojas, las cuales presentaron los valores más altos en el tratamiento de fertilización con gallinaza sin compostar, seguido muy de cerca por el tratamiento de fertirrigación, lo que evidencia la influencia de la disponibilidad de nutrientes en formas rápidamente asimilables para la planta sobre esta variable.

La mayor rentabilidad lograda por el tratamiento con químico, se debe básicamente a la menor cantidad de mano de obra empleada en la aplicación del fertilizante (3 jornales frente a 16 jornales para la aplicación de gallinaza), como al menor valor en el rubro pagado por el fertilizante (44,5 \% menos) en comparación por el equivalente en abono gallinaza sin compostar.

Los valores de baja rentabilidad para el tratamiento fertirriego, se debieron a los mayores costos directos en que se incurre, por la compra del equipo necesario para su implementación, como es el micro-controlador, electroválvulas y las mangueras de riego por goteo y que se depreció a 10 años.

La baja rentabilidad en el caso del tratamiento con abono compostado, se debió básicamente, al mayor costo de este tipo de abono comparado con la gallinaza sin compostar, a que se emplea el mismo número de jornales para su aplicación, y a su efecto desfavorable sobre la producción, debida seguramente a una menor disponibilidad de nutrientes para la planta comparado con las otras fuentes fertilizantes, pero que tiene un efecto muy positivo sobre la salud del suelo, al no promover la aparición de moscas y de otros tipos de organismos indeseados.

\section{CONCLUSIONES}

Las mayores producciones del cultivo de la cebolla de rama, se obtuvieron con la aplicación de gallinaza sin compostar, seguido muy de cerca por el tratamiento de fertirriego; lo que hace pensar, que con un mejor ajuste en los niveles de los nutrientes empleados en la dosificación del fertirriego de acuerdo con las diferentes épocas de cultivo, es factible superar los rendimientos obtenidos con la gallinaza si compostar, evitando los graves problemas al medio ambiente que este tipo de abono está causando y dando una salida a los productores de la zona frente a las regulaciones del ICA para el uso de gallinazas no compostadas.

En cuanto a la variable longitud de los seudotallos, los tratamientos de fertilizantes no presentaron ninguna diferencia estadística entre ellos, esto significa que este carácter de calidad de la cebolla rama, está determinado más por la profundidad efectiva del suelo, que permite una mejor labor de aporque de las plantas, y esta práctica motiva el alargamiento de los seudotallos.

En cuanto a la variación del diámetro de los seudotallos, los resultados indican que es un carácter poco influenciado por el ambiente y manejado por pocos genes, pero que puede ser mejorado mediante un incremento en los niveles de fertilización en los otros tratamientos. 
El análisis económico de los tratamientos sobre la producción de la cebolla de rama, bajo las condiciones del ensayo, determinó que se obtiene la mayor rentabilidad $(34,0 \%)$ con la fertilización química en forma sólida aplicada al suelo, razón de peso para que los productores abandonen el uso de gallinaza sin compostar, como fuente principal de nutrientes para el cultivo.

\section{BIBLIOGRAFÍA}

Agúdelo, T.C. 2007. Propuesta para el manejo y control integrado de la mosca domestica (Musca domestica) en el casco urbano del municipio de Guática Departamento de Risaralda. Tesis de pregrado, Universidad Tecnológica de Pereira, Pereira, Colombia.

Benavides, C. y Siles, G. 1990. Efectos de diferentes niveles de nitrogeno, fraccionamiento y momentos de aplicación sobre el crecimiento y rendimiento del maiz (Zea maiz L), var NB-6. Tesis de pregrado, Universidad Nacional Agraria, Managua, Nicaragua.

Bralts, V., Edwards, D. y Wu, I. 1987. Drip irrigation design and evaluation based on stadistical uniformity concept. Advances in irrigation 4: 67-117.

CARDER. 2008. Plan de ordenación y manejo de la cuenca hidrográfica del río Otún. Pereira, Colombia.

Castellanos, P. 1999. Manejo integral del cultivo de la cebolla de rama para el departamento de Risaralda. Tesis de Maestría, Universidad de Caldas, Facultad de Ciencias Agropecuarias, Manizales, Colombia.

CORPOICA. 1993. Diagnóstico Tecnológico Integral, Regional Nueve. Manizales, Caldas, Colombia.
Guerrero, A. 1996. El suelo, los abonos y la fertilizacion de los cultivos. Mundi - Prensa, Madrid- Barcelona, España.

Guzman, S. y Palacios, M. 2009. Instrumentos de política para gestión de servicios ecosistemicos en agorecosistemas cebolleros de la cuenca del río Otún. Recursos Naturales y Ambiente (58): 51-58.

Hagin, J., Sneh, M., y Lowengart, A. 2002. Fertigation Fertilization throughirrigation. En: Johnston, E.A., Editor. IPI Research Topics 23. International Potash Institute, Basel, Switzerland.

Jaramillo, J., Palacios, V. y Osorio, J. 1996. Aspectos generales de la producción de ajo y cebolla. En: López, V., Editor. El cultivo del ajo y las cebollas en Colombia. CORPOICA, PRONATTA, ICA. Santa Fe de Bogotá, Colombia.

Jaramillo, R.A. 1997. Climatologia de las regiones Andinas de Colombia. Federación Nacional de Cafeteros de Colombia, CENICAFE. Chinchina, Colombia.

Machado, M.O., Da Silva, G.A., Turatti, A., Pauletto, E. y Silveira, P. 1983. Efeito da adubação orgânica e mineral na produção do arroz irrigado e nas propriedades químicas e físicas do solo de Pelotas. Pesquisa Agropecuária Brasileira 18(6): 583-591.

Silva, N., Ramon, L.R., Modesto, D.B., Nogueira, E.M., Martínez, M., Fandiño, J., Cancela y Rey, B. 2013. Rango de presión en emisores de riego en ladera en viñedo. XXXI Congreso Nacional de Riegos, C-17., Orihuela.

Vallejo, F. y Estrada, E. 2002. Mejoramiento genetico de plantas. Universidad Nacional de Colombia Sede Palmira, Palmira, Colombia.

Fecha de recepción: 25/05/2016

Fecha de aceptación: 13/06/2017

Publicado en línea: 22/06/2017

Para citar este artículo: Polanco-Puerta, M.F. y Gómez-Posada, S. 2017. Evaluación de tres programas de fertilización edáfica en el cultivo de la cebolla de rama en la cuenca media del rio Ótun. Revista Intropica Vol. 12(1): 31 - 40. DOI: http://dx.doi.org/10.21676/23897864.2203 